\title{
Improving Administration of Fascia-iliaca Blocks for Patients With a Fractured Neck of Femur by Clinical Audit
}

Isaac Okereke ( $\sim$ okereke.isaac@gmail.com )

The Royal London Hospital https://orcid.org/0000-0001-8862-6419

Sridhar Rao Sampalli

Salisbury Hospital NHS Trust: Salisbury NHS Foundation Trust

\section{Research Article}

Keywords: Fascia iliaca block, audit, neck of femur fracture, anaesthesia

Posted Date: May 13th, 2021

DOI: https://doi.org/10.21203/rs.3.rs-502228/v1

License: (c) (i) This work is licensed under a Creative Commons Attribution 4.0 International License.

Read Full License 


\section{Abstract}

\section{Background}

Hip fracture is the most common serious injury in older people. It is also the most common reason for older people to need emergency anaesthesia and surgery, and the commonest cause of death following an accident. A FICB is the injection of anaesthetic agents into the fascia- iliaca compartment with the effect of blocking the lumbar plexus via an anterior approach. FICB is clinically safe and efficient and provides consistent analgesic effects irrespective of the performing doctor's experience of frailty fractures of the proximal femur.

\section{Methods}

Data from the National Hip Fracture Database (NHFD) for all patients admitted with a neck of femur fracture between October 2018 and May 2019 was interrogated and audited. Results of this audit were discussed in the department of Trauma \& Orthopaedics' and the Trust's mortality review meetings. Teaching sessions were held for doctors and filling out of the neck of femur fracture proforma to detail administration or not of FICB and a valid reason when the later occurred was encouraged. A re-audit was carried out in May 2020 where a retrospective study of patients admitted with a neck of femur fracture over six months from October 2019 to April 2020 was done to assess improvement in compliance rates of administration of fascia iliaca blocks.

\section{Results}

We noted a statistically significant increase in the number of patients who got a fascia iliaca block on presentation with a fractured neck of the femur from after our second audit $(p<0.00001)$. There were no complications associated with the administration of FICB to patients with neck of femur fractures.

\section{Conclusion}

This study showed that clinical processes could be improved through audits, staff education and by employing the use of proformas to ensure compliance.

\section{Introduction}

A hip fracture is the most common serious injury in older people. It is also the most common reason for older people to need emergency anaesthesia and surgery and the commonest cause of death following an accident (1). With a mortality rate of $10 \%$ at one month and between $20-35 \%$ at one year after injury, fractures around the hip account for more than 1 in 45 of all hospital beds in England and Northern Ireland, and about 1 in 33 in Wales. Only a fraction of patients who have suffered a fracture to their hip return to baseline function, with most becoming dependent and needing long term care, thereby costing the NHS about $£ 1$ Billion annually, which is about $1 \%$ of the annual NHS budget. 
Standardised mortality ratios show mortality to be much higher in people after a fractured neck of the femur than in the general population of comparable age, remaining raised for many months after fracture. The persistently increased standardised mortality ratio may indicate continuing sequelae of the fracture or that people fracturing their neck of the femur are frailer and more ill than the general population of similar age (2).

Most patients who present with fractures around the hip are often elderly, frail, and have several comorbidities and are consequently polypharmacy. $50-70 \%$ of elderly patients who have sustained hip fractures report severe to very severe pain in the first 24 hours post-injury. The obvious fracture and the excruciating pain will place limits on their ability to move. This negatively impacts pressure area care, food and fluids ingestion, and good sleep, not to mention the body's neuroendocrine responses and compromised pulmonary function as a sequential reaction to pain (3). At rest, one-third of patients with a proximal femur fracture will have mild or no pain, one-third will have moderate pain, and one-third will have severe pain. Extracapsular fractures are more painful than intracapsular fractures due to the greater degree of periosteal damage (4).

The assessment and management of pain in these patients can be challenging due to several reasons: increased incidence of complications such as gastrointestinal bleed and renal injuries from NSAIDS use, respiratory depression, and constipation from Opioid use, as well as difficulty in pain assessment due to co-existing cognitive impairment in a lot of cases. Effective pain management in these patients leads to improved outcomes post-operatively and reduces postoperative delirium and pulmonary complications.

The NICE guidelines for managing hip fractures recommend that clinicians consider adding nerve blocks if paracetamol and opioids do not provide sufficient preoperative pain relief or limit opioid dosage.

Furthermore, nerve blocks should be administered by trained personnel (5).

Dalens et al. first described fascia iliac compartment blocks (FICB) in paediatric patients in 1989 (6). A FICB is the injection of anaesthetic agents into the fascia- iliaca compartment with the effect of blocking the lumbar plexus via an anterior approach (7). FICB is clinically safe and efficient and provides consistent analgesic effects irrespective of the performing doctor's experience of frailty fractures of the proximal femur (8).

\section{Aims and Objectives}

In this study, we hypothesised that the number of patients receiving FICB for a neck of femur fracture could be improved by audits, educational/teaching sessions, and employing a proforma, in line with the NICE Guidelines.

\section{Methods}

Data from the National Hip Fracture Database (NHFD) for all patients admitted with a neck of femur fracture between October 2018 and May 2019 was interrogated and audited. The number of patients receiving FICB, reasons for non- administration of a block, and presentation time in the Accident \& 
Emergency department were recorded. 148 were reviewed, 75\% $(n=111)$ females; mean age 84.38years (SD 7.66).

After the first audit, results were discussed in the department of Trauma \& Orthopaedics' and the Trust's mortality review meetings. A decision for all patients with necks of femur fractures to receive a fascia iliac block in the Emergency Department except when contraindicated. Measures were taken to improve compliance with the administration of FICB by implementing small one-to-one fascia iliaca block teaching sessions for Emergency Department and Trauma \& Orthopaedics junior doctors. This aimed to instil a culture of routine administration of blocks by all junior doctors admitting neck of femur fractures to reduce the over-reliance on opiates for pain control, reducing unwanted and potentially debilitating side effects. Proper filling out of the neck of femur proforma was encouraged to ensure FICB were signed off by administering doctors and a reason documented when contra-indicated.

In May 2020, we undertook a retrospective audit of patients admitted with a neck of femur fracture over six months from October 2019 to April 2020 was undertaken to assess compliance in administration of fascia iliaca blocks. The data was again extracted from the National Hip Fracture Database and scanned notes of patients to determine FICB administration records, reasons for non-administration and pain scores. Also, patient demographics and time of presentation at the Accident \& Emergency department were recorded. A total of 169 patients' records were reviewed. There were $69 \%(n=117)$ females; mean age of 82years (SD 7.2). All statistical analysis was performed using SPSS (version 25.0; SPSS Inc, Chicago, IL). The results of each audit were compared and statistically analysed using the student's t-test to compare continuous variables and the chi-square test for dichotomous variables. A p-value of $<0.05$ was considered statistically significant.

\section{Ethical statement}

The authors conducted a retrospective audit; as such, there was no additional patient contact and no requirement for formal ethical approval. The project was registered with the department's clinical governance department and was conducted per the Declaration of Helsinki and useful clinical practice guidelines.

\section{Results}

Table I. summarizes results pre-and post- interventions. $24.5 \%(n=45)$ received a fascia iliaca block on admission, compared to $44.3 \%(n=94)$ who received blocks in the subsequent audit. We noted a statistically significant increase in the number of patients who got a fascia iliaca block on presentation with a fractured neck of the femur from after our second audit $(p<0.00001)$. 
Table I.

\begin{tabular}{|llll|}
\hline & $\begin{array}{l}\text { First Audit } \\
(\boldsymbol{n = 1 8 4})\end{array}$ & $\begin{array}{l}\text { Second Audit } \\
(\boldsymbol{n}=\mathbf{1 6 9 )}\end{array}$ & $\boldsymbol{p}$ value \\
\hline Gender $m: f$ & $1: 3$ & $1: 2.25$ & $0.25412^{\star}$ \\
\hline Mean age $(y r s)$ & $84.38 \pm 7.66$ & $82 \pm 7.2$ & $0.0029^{\star \star}$ \\
\hline FICB administered $n(\%)$ & $45(24.5 \%)$ & $94(44.3 \%)$ & $<0.00001^{\star}$ \\
\hline Mean pre- FICB pain score & 8.9 & 9.5 & $0.2316^{\star \star}$ \\
\hline Mean post- FICB pain score & 4 & 3.6 & $<0.0011^{\star \star}$ \\
\hline Reason for non-administration documented & 5 & 3 & $0.56122^{\star}$ \\
\hline
\end{tabular}

*Chi-square ** T-test

FICB, fascia-iliaca compartment block

A large proportion of patients in this audit presented to ED between 08:00 and 19:59, as shown in Figure I when there are usually more hands in the department and more senior staff on duty than on the night shifts. There were no documented local or systemic complications to fascia-iliaca blocks in both audits.

\section{Discussion}

Patients who present with fractures of the neck of the femur are usually in very considerable and intractable pain. Management of this pain by conventional analgesia is complicated by side effects leading to an extended hospital stay and generally poorer outcomes.

Pain from fractures of the femoral neck is carried by the obturator, sciatic and femoral nerves in accordance with Hilton's law. The administration of fascia iliaca blocks for a neck of femur fractures is a safe, simple and rapidly effective pain management method that reduces the need for systemic analgesia and provides consistent simultaneous blockade of the lateral cutaneous femoral and femoral nerves (9). It is a technique that can be taught with minimal instruction and can be performed by junior staff (10). Stevens et al. demonstrated that patients who underwent a FICB used significantly less morphine over 24 hours than the control group, which used morphine alone. Furthermore, FICB has a duration of effect of about 8-10hours following a single block (11).

All doctors involved in managing neck of femur fractures should be trained on administering fascia iliaca blocks. This should form part of the protocol for admissions of frailty fractures around the proximal femur.

Contraindications to a FICB are:

- An uncooperative patient 
- A known hypersensitivity reaction to local anaesthesia

- A neurological problem affecting the fractured limb

- Presence of local infection

- Previous femoral bypass surgery on ipsilateral limb

- Patient currently on anticoagulation

This study showed that clinical processes could be improved through audits, staff education, and using proformas to ensure compliance.

There are some limitations to this study. Our data did not include the number and grades of junior doctors who received teaching on FICB administration. This is important as most junior doctors spend about 6 months in each posting. The duration between our first and second audits implies that a different set of junior doctors would have been working in the various departments.

In conclusion, this study showed the effectiveness of clinical audit against standards, with feedback, in modifying practice amongst healthcare professionals and improving patient care.

\section{Declarations}

- Ethics approval and consent to participate: Not applicable

- Consent for publication: Not applicable

- Availability of data and materials: The datasets generated and/or analysed during the current study are available in the Salisbury District Hospital NHS, NHFD.

- Competing interests: The authors declare that they have no competing interests

- Funding: Not applicable

- Author's Contributions: 10 conceptualized this study, did the literature review, and wrote the first draft of manuscript. SS proofread and approved final manuscript. The first draft of the manuscript was written by Isaac Okereke and both authors commented on previous versions of the manuscript. All authors read and approved the final manuscript.

- Acknowledgements: Not applicable

\section{References}

1. Royal College of Physicians. National Hip Fracture Database (NHFD) | RCP London [Internet]. 2020 [cited 2021 May 5]. Available from: https://www.rcplondon.ac.uk/projects/national-hip-fracturedatabase-nhfd

2. Goldacre MJ, Roberts SE, Yeates D. Mortality after admission to hospital with fractured neck of femur: Database study. Br Med J [Internet]. 2002 Oct 19 [cited 2021 May 5];325(7369):868-9. Available from: http://www.bmj.com/ 
3. Welcome to the website of Mid and South Essex NHS Foundation Trust | Mid and South Essex NHS Foundation Trust [Internet]. [cited 2021 May 5]. Available from: https://www.mse.nhs.uk/

4. Dixon J, Ashton F, Baker P, Charlton K, Bates C, Eardley W. Assessment and Early Management of Pain in Hip Fractures: The Impact of Paracetamol. Geriatr Orthop Surg Rehabil [Internet]. 2018 Jan 1 [cited 2021 May 5];9:215145931880644. Available from: /pmc/articles/PMC6202735/

5. National Institute for Health and Care Excellence (NICE). Overview | Hip fracture: management I Guidance | NICE. 2011 [cited 2021 May 5]; Available from: https://www.nice.org.uk/guidance/cg124

6. Dalens B, Vanneuville G, Tanguy A. Comparison of the fascia iliaca compartment block with the 3-in1 block in children. Anesth Analg [Internet]. 1989 [cited 2021 May 5];69(6):705-13. Available from: https://oce.ovid.com/article/00000539-198912000-00003/HTML

7. Mostafa SF, Eid GM, Elkalla RS. Patient-controlled fascia iliaca compartment block versus fentanyl patient-controlled intravenous analgesia in patients undergoing femur fracture surgery. Egypt $\mathrm{J}$ Anaesth [Internet]. 2018 Jan 1 [cited 2021 May 5];34(1):9-13. Available from: https://www.tandfonline.com/action/journallnformation?journalCode=teja20

8. Fujihara Y, Fukunishi S, Nishio S, Miura J, Koyanagi S, Yoshiya S. Fascia iliaca compartment block: Its efficacy in pain control for patients with proximal femoral fracture. J Orthop Sci. 2013 Sep 1;18(5):793-7.

9. Lopez S, Gros T, Bernard N, Plasse C, Capdevila X. Fascia iliaca compartment block for femoral bone fractures in prehospital care. Reg Anesth Pain Med [Internet]. 2003 May 1 [cited 2021 May 5];28(3):203-7. Available from: https://rapm.bmj.com/content/28/3/203

10. Haddad FS, Williams RL. Femoral nerve block in extracapsular femoral neck fractures. J Bone Jt Surg - Ser B [Internet]. 1995 Nov 1 [cited 2021 May 5];77(6):922-3. Available from: https://online.boneandjoint.org.uk/doi/abs/10.1302/0301-620X.77B6.7593107

11. Stevens M, G Harrison MM. Modified fascia iliaca block in total hip arthroplasty: Anaesthesia and Intensive Care Journal [Internet]. ANAESTHESIA AND INTENSIVE CARE. 2017 [cited 2021 May 5]. Available from: https://aaic.net.au/document/?D=2007052

\section{Figures}




\section{Time of arrival in ED}

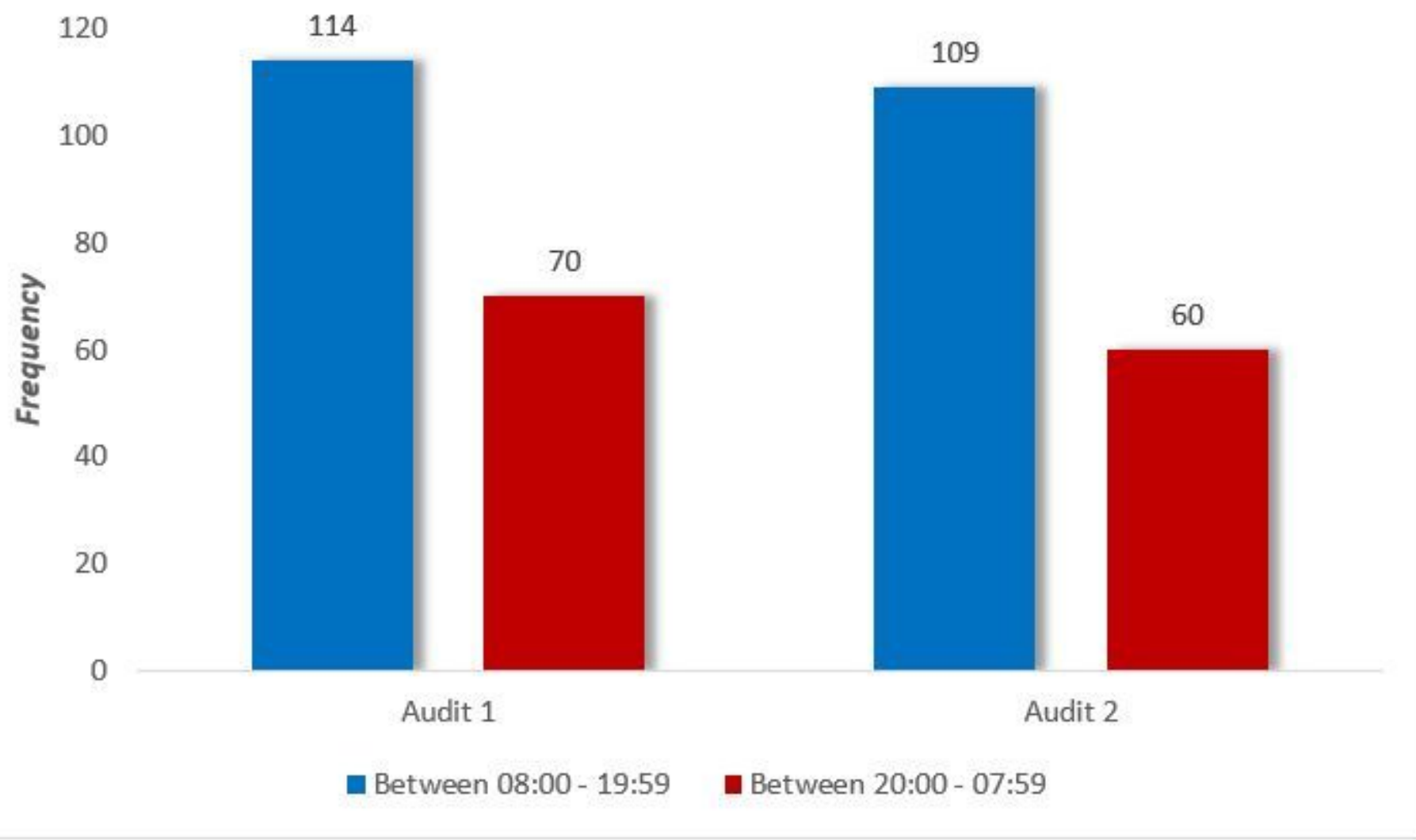

\section{Figure 1}

Time of arrival to the Emergency Department. 